\title{
Aortoiliac Occlusive Disease as a Cause of Allograft Kidney Dysfunction and Refractory Hypertension
}

\author{
HaengJin OHE \\ Department of surgery, Seoul Paik hospital, Inje University, Korea
}

\begin{abstract}
Aortoiliac occlusive disease (AIOD), especially proximal to the transplant artery, in kidney transplant patient activates the renin-angiotensin-aldosterone system by limiting graft renal perfusion and causes symptoms that can occur with transplant renal artery stenosis (TRAS) such as refractory hypertension, water retention, and graft renal dysfunction. Immediate clinical suspicion is difficult due to the nature of the progressive disease unlike TRAS. Herein, we present an interesting case of bilateral common iliac artery occlusion (AIOD, TASC II, type C) that manifested as uncontrolled blood pressure and decreased allograft function in a patient who had kidney transplant 17 years ago. The patient was successfully diagnosed with duplex scan, ankle-brachial index (ABI) and computed tomography angiography and treated with percutaneous luminal angioplasty and stent graft insertion.
\end{abstract}

Key Words: Allograft dysfunction, Common iliac artery, Kidney transplantation, Refractory hypertension

Aortoiliac occlusive disease (AIOD) is a peripheral arterial disease caused by atherosclerosis from the infra-renal artery to the iliac arteries. AIOD in renal transplant patients may limit the blood flow to the graft, resulting in symptoms and signs similar to Transplant renal artery stenosis (TRAS). Considering that atherosclerosis causes gradual vascular occlusion, it is necessary to distinguish it from TRAS.

Herein, we present a case of AIOD diagnosed in 17 years after transplantation with graft kidney dysfunction and refractory hypertension and treated with percutaneous angioplasty and stent graft insertion.

\section{CASE}

19 years ago, a 49-year-old woman underwent a non-related live kidney transplant for an unknown caused end-stage renal disease. For 14 years after the transplantation, there was no rejection and complications, and remained stable graft function in the baseline serum creatine of $0.8-1.1 \mathrm{mg} / \mathrm{dL}$. There was a temporary rise in serum creatine at $1.97 \mathrm{mg} / \mathrm{dL}$ at 15 years and $1.64 \mathrm{mg} / \mathrm{dL}$ at 16 years postoperatively, but it stabilized soon after short-term intravenous saline administration. The baseline serum creatine was then maintained at $1.2-1.5 \mathrm{mg} / \mathrm{dL}$,
Corresponding Author: HaengJin OHE, Department of Surgery, Seoul Paik hospital, 9, mareunae-ro, Jung-gu, Seoul 04551, Korea

Tel: +82-02-2270-0247 Fax: +82-02-2270-0373 E-mail: Jin.theDoc@gmail.com

\section{(c) $1(3)$}

Articles published in Kosin Medical Journal are open-access, distributed under the terms of the Creative Commons Attribution Non-Commercial License (http://creativecommons.org/licenses/by-nc/4.0/) which permits unrestricted non-commercial use, distribution, and reproduction in any medium, provided the original work is properly cited.
Received: Nov. 05, 2019

Revised: Nov. 05, 2019

Accepted: Nov. 22, 2019 
slightly higher than before, and the systolic blood pressure was maintained at $140 \mathrm{mmHg}$ by taking four different type of antihypertensive drugs twice daily.

At 17 years postoperatively, the patient was hospitalized due to a sharp decrease in urine volume, general edema, shortness of breath, and an increase in serum creatine of $3.65 \mathrm{mg} / \mathrm{dL}$. Duplex ultrasonography showed no change of the appearance and echogenicity of the graft kidney, but was measured by the pulsus parvus et tardus pattern and the reduced resistive index(RI) of 0.5 by the intrarenal artery doppler scan (Fig. 1). Suspecting graft kidney ischemia, CT angiography was performed to identify the site of stenosis. CT angiography presented bilateral common iliac artery stenosis (TASC classification C) (Fig. 2). The patient had no symptom of claudication, but performed an ankle-brachial index to quantify the degree of stenosis before and after treatment. The result of ABI was 0.73 on the right side and 0.50 on the left side of the transplanted
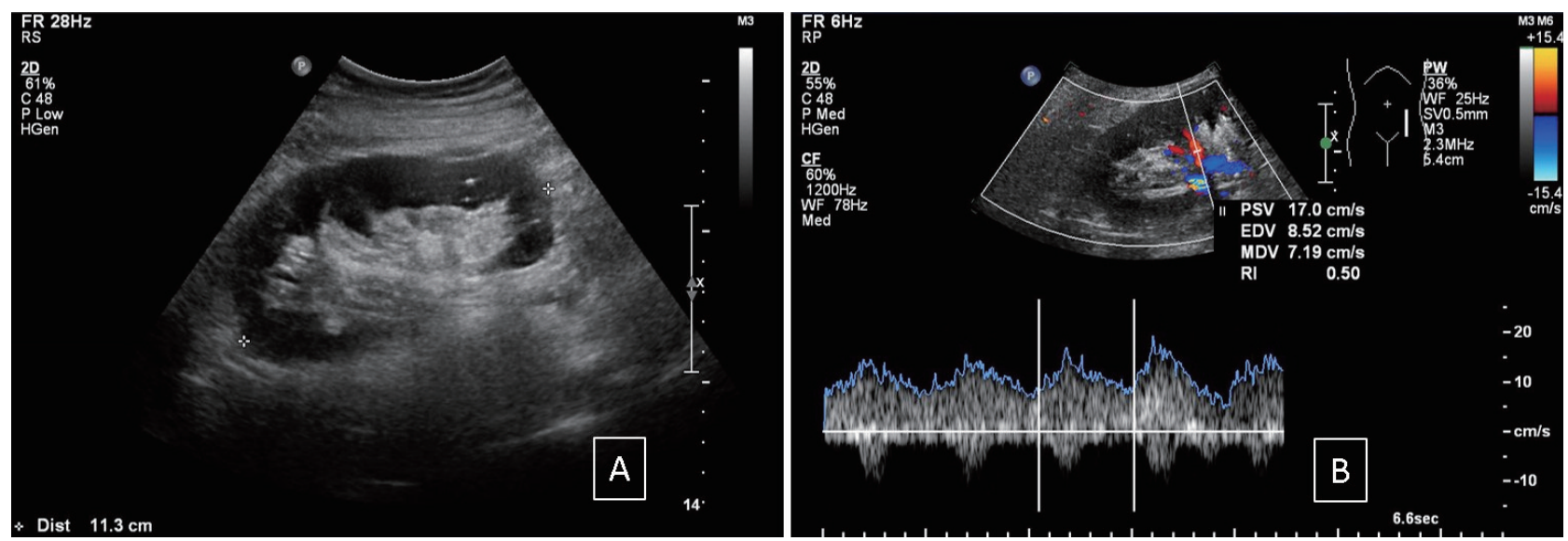

Fig. 1. Duplex ultrasonography showing (A) normal size and echogenicity of graft kidney and (B) low resistive index and pulsus parvus et tardus pattern in the intrarenal artery
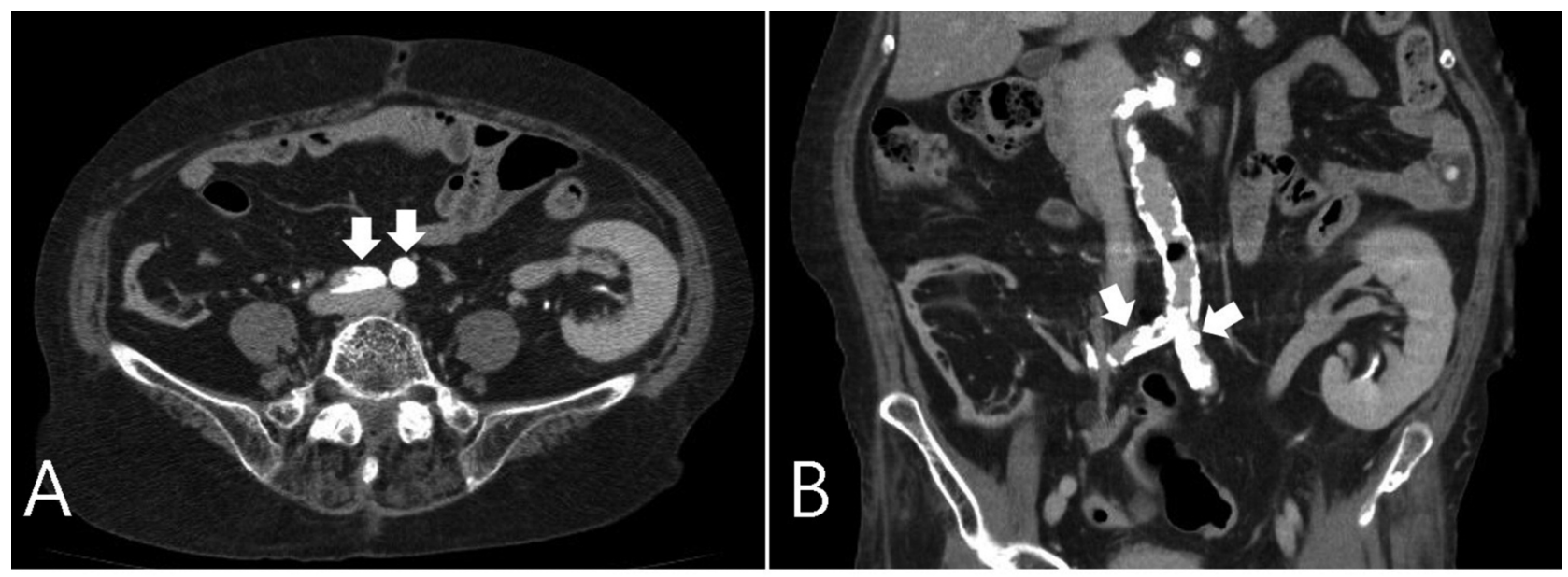

Fig. 2. Non-contrast-enhanced Computed Tomography angiography showing heavily calcified chronic total occlusion of both common iliac artery (white arrows). (A)axial scan and (B)coronal scan 
kidney (normal value: 0.9-1.2).

Under local anesthesia, both common femoral artery approach was performed, and initial angiography was obtained via less stenotic lesion of Rt. access. The lesion of Lt. common iliac was neartotal occlusion, and the stenosis of right common iliac artery was up to $80 \%$ of the lumen. Upon the left approach, successful retrograde recanalization was done with .014 " guide wire and it's supporting catheter. Pre-ballooning $(3.5 * 20$ mm, PTCA balloon, Nimbus PICO, BARD) was performed to smoothly deliver stent grafts to the lesion, and balloon expandable stent grafts $(9 *$ $58 \mathrm{~mm}$, Lifestream, BARD, 9 ATM) was inflated until the patient complained of pain. The lesions on the right also was deployed stent graft of the same size and length. The procedure was had completed after confirming patent artery of graft kidney limb without thromboembolism (Fig. 3). The next day, ABI rose to 0.95 on the right and 0.89 on the left.

Six months after the procedure, the serum creatine level decreased to $0.97 \mathrm{mg} / \mathrm{dL}$, and one antihypertensive drug was enough to make her blood pressure stable. Doppler waveform and resistance index of intra-renal artery were normalized (Fig. 4).

\section{DISCUSSION}

AIOD is a peripheral artery disease in which gradual occlusion of arterial blood due to atherosclerosis, which extends from the infra-renal aorta to the iliac arteries, limits blood flow to the lower extremities. Trans Atlantic Inter-Society

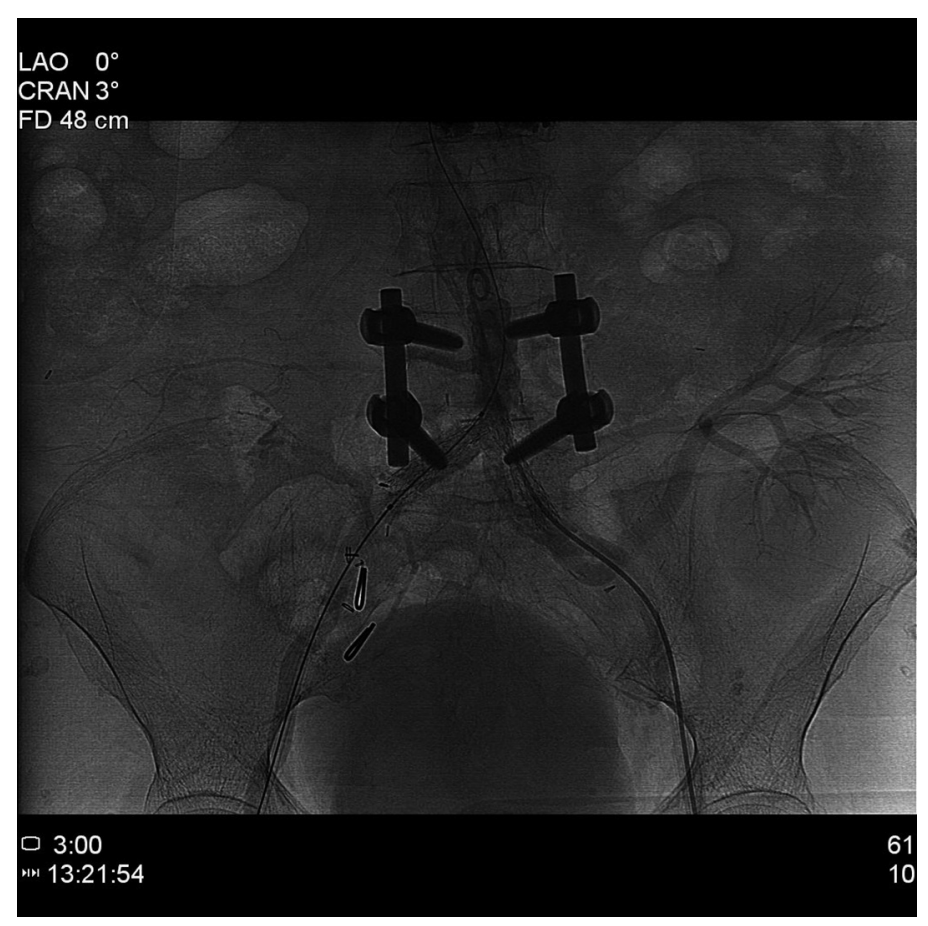

Fig. 3. Completing angiography showing successful deployment of stent grafts in both common iliac artery occlusions without graft kidney thromboembolism 


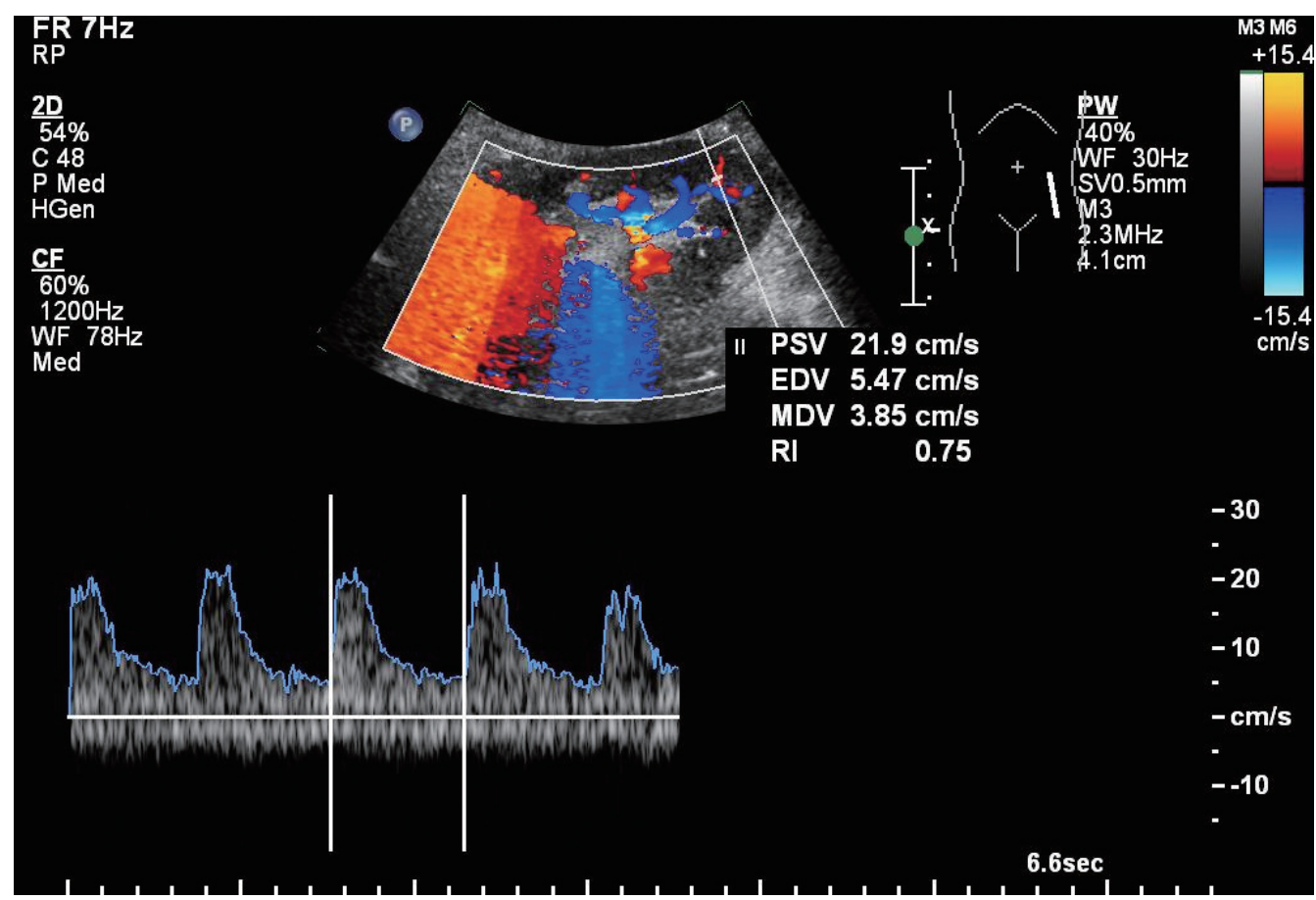

Fig. 4. Follow-up Duplex ultrasonography showing normal resitive index and flow pattern in intrarenal artery

Consensus Group (TASC), published by European and North American vascular specialists, has issued recommendations for the treatment and management of peripheral arterial disease. The proposed Trans-Atlantic Inter-Society Consensus (TASC II) guidelines are now widely used. ${ }^{1}$ TASC guideline states that TASC classification A, B are appropriate for interventional treatment, TASC classification C, D recommend surgical treatment. However, in the TASC classifications $\mathrm{C}$ and $\mathrm{D}$, interventional procedures have been applied depending on the severity of the patient's comorbidity and on the patient's own choice. Since the publication of the TASC II Guideline in 2007, the development of interventional instruments and techniques has led to an increasing number of interventions as primary treatments in low risk groups. In low-groups, interventions are often used as the primary treat- ment. ${ }^{2}$ Recent COBEST study conducted in 2016 suggested the use of stent grafts in TASC classification $\mathrm{C}$ and $\mathrm{D}$ lesions. ${ }^{3}$

AIOD in renal transplant patients is a progressive obstruction of the recipient's artery due to atherosclerosis and should be distinguished by timing and lesion location from the TRAS that occurs at the anastomotic site at 6 months postoperatively. ${ }^{4}$ Considering the 15-20 year lifespan of the graft, a decrease in graft function due to gradual closure of inflow artery may be underestimated as a natural graft kidney failure. ${ }^{5}$

If the inflow of the transplant kidney is restricted due to AIOD, the renin-angiotensin-aldosterone system may be activated, resulting in symptoms and signs of refractory hypertension, water retention, and elevated creatine in the blood. However, the gradual development of the collateral circulation into the lower extremities may not ap- 
peal to the symptoms of intermittent claudication. ${ }^{6}$ Some groups emphasize that refractory hypertension due to graft kidney ischemia is the main indication of disease. ${ }^{7}$

Duplex ultrasonography should be the first choice in screening and then angiography should be performed to confirm a location of the lesion. ${ }^{4}$ Because of the heavy calcification of the lesion, CT angiography without the use of contrast media can also diagnose a location of the lesion. $\mathrm{ABI}$ is a non-invasive screening test for peripheral arterial disease, which is relatively accurate and economical indirectly assessing the vascular status of patients. ABI for diagnosing peripheral arterial disease is $80 \%$ or higher in sensitivity and specificity. A number of guidelines suggested that further diagnostic tests are needed to differentiate peripheral arterial disease when ABI is less than 0.9. It can also be useful for comparison before and after treatment and follow-up. ${ }^{8}$

\section{REFERENCES}

1. Norgren L, Hiatt WR, Dormandy JA, Nehler MR, Harris KA, Fowkes FG, et al. Inter-Society Consensus for the Management of Peripheral Arterial Disease (TASC II). Eur J Vasc Endovasc Surg 2007;33 Suppl 1:S5-75.

2. Bracale UM, Giribono AM, Spinelli D, Del Guercio L, Pipitò N, Ferrara D, et al. Longterm Results of Endovascular Treatment of TASC C and D Aortoiliac Occlusive Disease with Expanded Polytetrafluoroethylene Stent Graft. Ann Vasc Surg 2019;56:254-60.
3. Mwipatayi BP, Sharma S, Daneshmand A, Thomase SD, Vijayan V, Altaf N, et al. Durability of the balloon-expandable covered versus bare-metal stents in the Covered versus Balloon Expandable Stent Trial (COBEST) for the treatment of aortoiliac occlusive disease. J Vasc Surg 2016;64:83-94 e1.

4. Bruno S, Remuzzi G, Ruggenenti P. Transplant renal artery stenosis. J Am Soc Nephrol 2004;15:134-41.

5. Coemans M, Süsal C, Döhler B, Anglicheau D, Giral M, Bestard O, et al. Analyses of the short- and long-term graft survival after kidney transplantation in Europe between 1986 and 2015. Kidney Int 2018;94:964-73.

6. Becker BN, Odorico JS, Becker YT, Leverson G, McDermott JC, Grist T, et al. Peripheral vascular disease and renal transplant artery stenosis: a reappraisal of transplant renovascular disease. Clin Transplant 1999;13:349-55.

7. Voiculescu A, Hollenbeck M, Plum J, Hetzel GR, Mödder U, Pfeiffer T, et al. Iliac artery stenosis proximal to a kidney transplant: clinical findings, duplex-sonographic criteria, treatment, and outcome. Transplantation 2003;76:332-9.

8. Misra S, Shishehbor MH, Takahashi EA, Aronow HD, Brewster LP, Bunte MC, et al. Perfusion Assessment in Critical Limb Ischemia: Principles for Understanding and the Development of Evidence and Evaluation of Devices: A Scientific Statement From the American Heart Association. Circulation 2019;140:e657-72. 